\title{
Stress myocardial blood flow and revascularization in chronic coronary artery disease
}

\author{
Teemu Maaniitty, MD, PhD, a,b Juhani Knuuti, MD, PhD, a and Antti Saraste, MD, \\ $\mathrm{PhD} \mathrm{D}^{\mathrm{a}, \mathrm{c}}$ \\ a Turku PET Centre, Turku University Hospital and University of Turku, Turku, Finland \\ b Department of Clinical Physiology, Nuclear Medicine and PET, Turku University Hospital, Turku, \\ Finland \\ c Heart Center, Turku University Hospital and University of Turku, Turku, Finland
}

Received May 4, 2021; accepted May 4, 2021

doi: 10.1007/s12350-021-02687-7

Key Words: Positron emission tomography $\cdot$ Radiowater $\cdot$ Chronic coronary syndromes • Myocardial ischemia $\cdot$ Myocardial blood flow

See related article, pp. 1887-1899

Positron emission tomography (PET) myocardial perfusion imaging (MPI) using oxygen-15-labeled water $\left({ }^{15} \mathrm{O}\right.$-water) has been increasingly used in the evaluation of coronary artery disease (CAD). ${ }^{15} \mathrm{O}$-water has favorable kinetics for absolute quantification myocardial blood flow (MBF) that can be presented as parametric maps of stress MBF or myocardial flow reserve (MFR) enabling evaluation of regional perfusion abnormalities. ${ }^{1}$ Prospective clinical studies have shown that both stress MBF and MFR assessed by ${ }^{15} \mathrm{O}$-water PET have high diagnostic accuracy in the detection of significant CAD defined as an abnormal fractional flow reserve (FFR) in patients with suspected CAD. ${ }^{2-4}$ In addition to diagnostic performance, clinical value of a diagnostic test depends on its ability to stratify patients at low or high risk of adverse events and impact on choosing subsequent treatment strategy.

The study by Promteangtrong et al. published in this issue of the Journal of Nuclear Cardiology presents a detailed evaluation of the impact of ${ }^{15} \mathrm{O}$-water PET

Reprint requests: Antti Saraste, MD, PhD, Heart Center, Turku University Hospital and University of Turku, Hämeentie 11, 20520

Turku, Finland; antti.saraste@utu.fi

J Nucl Cardiol 2022;29:1900-2.

$1071-3581 / \$ 34.00$

Copyright $\Subset 2021$ American Society of Nuclear Cardiology. results on referral to invasive coronary angiography (ICA) and performance of coronary revascularization in a retrospective cohort of 57 patients undergoing a clinically indicated myocardial perfusion study. ${ }^{5}{ }^{15} \mathrm{O}$-water PET showed normal myocardial perfusion in 18 (32\%) patients of whom only one underwent downstream ICA (showing non-obstructive coronary atherosclerosis) within 6 months, resulting in an ICA referral rate of $6 \%$. ${ }^{15} \mathrm{O}$-water PET was defined abnormal in the presence of at least one coronary territory having reduced stress $\operatorname{MBF}(\leq 2.3 \mathrm{~mL} / \mathrm{g} / \mathrm{min})$ or reduced MFR $(\leq 2.5)$ based on the previously reported cut-off values. ${ }^{1-4}$ Compared with normal stress MBF or MFR, a substantially higher ICA referral rate of $49 \%$ was found among patients with abnormal findings. Out of the 19 patients having reduced stress MBF or MFR referred to ICA, 12 patients subsequently underwent coronary revascularization resulting in actual revascularization rate of $63 \%$. Interestingly, global and regional stress MBF and MFR provided very similar predictive accuracies. These findings indicate that the results of ${ }^{15} \mathrm{O}$-water PET MPI were strongly associated with subsequent referral to ICA and revascularizations.

The findings by Promteangtrong et al. in a small cohort of patients are in line with previously published studies regarding patient management after ${ }^{15} \mathrm{O}$-water PET MPI. ${ }^{6,7}$ In 375 patients with suspected CAD, Danad et al. analyzed referral to ICA and revascularizations within a 60-day time period after hybrid imaging combining coronary computed tomography angiography (CTA) and ${ }^{15} \mathrm{O}$-water PET MPI. ${ }^{6}$ Downstream ICA 
referral rate was $5 \%$ and no revascularizations were performed in the absence of obstructive CAD on coronary CTA, whereas stenosis $(\geq 50 \%)$ by coronary CTA combined with abnormal PET MPI finding was associated with ICA referral rate of $88 \%$ and early revascularization rate of $72 \%$. In turn, Stenström et al. analyzed referral to ICA and revascularizations within 6 months after performing ${ }^{15} \mathrm{O}$-water PET to evaluate hemodynamic significance of suspected obstructive lesion identified by coronary CTA. ${ }^{7}$ A total of 670 symptomatic patients with suspected CAD first underwent coronary CTA, and those showing suspected obstructive stenosis on coronary CTA underwent stressonly ${ }^{15} \mathrm{O}$-water PET MPI. The ICA referral rates were $5 \%$ vs. $55 \%$ and early revascularization rates $17 \%$ (i.e., only a single patient) vs. $58 \%$ in patients with normal vs. abnormal stress MBF (i.e., stress $\mathrm{MBF} \leq 2.3 \mathrm{~mL} / \mathrm{g} / \mathrm{min}$ ), respectively.

In the study by Promteangtrong et al., no adverse cardiac events occurred in the conservatively treated patients during a short 6-month follow-up period. ${ }^{5}$ Longer-term outcomes have been analyzed by Stenström et al. demonstrating a low annual incidence rate $(0.3 \%)$ for the composite endpoint of non-fatal myocardial infarction or cardiovascular death in patients with either obstructive CAD excluded by coronary CTA alone or suspected obstructive stenosis but normal stress MBF during a median of 5.2 years. ${ }^{7}$ In line with quantitative data obtained using other perfusion tracers, ${ }^{1}$ recent studies have demonstrated that abnormal stress MBF or MFR assessed by ${ }^{15} \mathrm{O}$-water PET is indeed an independent predictor of myocardial infarction and death. ${ }^{8-10}$

The study of Promteangtrong et al. also provides an analysis on the diagnostic accuracy of ${ }^{15} \mathrm{O}$-water PET MPI. ${ }^{5}$ The results of this analysis should be interpreted with caution as it was restricted to the patients undergoing downstream ICA, therefore inducing bias. Furthermore, there are well-known discrepancies between anatomical and functional measures of stenosis severity, and the observed relatively low predictive value of abnormal MBF could be explained by the presence of microvascular dysfunction in the absence of obstructive epicardial CAD in a cohort including many patients with previously diagnosed CAD and high prevalence of risk factors for CAD. Finally, in per-vessel analysis, the discrepancies between PET MPI and ICA may be partly related to differences between individual and standardized coronary artery territories that were assumed in the perfusion analysis. In above-mentioned situations, non-invasive hybrid imaging combining anatomical coronary CTA data and functional perfusion data might be useful and improve specificity. ${ }^{1,4,11,12}$

Recently updated clinical guidelines for the diagnosis and management of chronic coronary syndromes recommend the use of either functional imaging of myocardial ischemia or coronary CTA as the initial noninvasive tests for diagnosing CAD in symptomatic patients. ${ }^{13}$ Furthermore, functional evaluation of ischemia is usually required before revascularization decisions, if the symptoms are not adequately relieved by optimal medical therapy alone or if there are highrisk findings. ${ }^{13}$ The study of Promteangtrong et al. demonstrates that treatment decisions adhered to the findings of ${ }^{15} \mathrm{O}$-water PET MPI in that invasive testing was deferred in patients with normal findings. ${ }^{5}$ However, a relatively large proportion of patients can be treated with medical therapy even in the presence of myocardial ischemia. Recently, also the results of the randomized ISCHEMIA trial have highlighted the relatively good prognosis of patients with chronic coronary artery disease on optimal medical therapy in the presence or absence of revascularization procedures. ${ }^{14}$ However, retrospective studies in large patient cohorts have shown an association between prognostic benefit obtained by revascularization and the severity of myocardial perfusion abnormalities assessed as extent of ischemia or reduction in MFR. ${ }^{15-17}$

Promteangtrong et al. should be congratulated on their detailed analysis of the associations between ${ }^{15} \mathrm{O}$ water PET MPI findings and downstream invasive testing as well as revascularization decisions in patients referred for evaluation of CAD in their center. ${ }^{5}$ Impact on subsequent choice of therapeutic strategy is an important measure of the value of a diagnostic technique, such as ${ }^{15} \mathrm{O}$-water PET. In the absence of prospective randomized trials, retrospective registry studies may shed light on the complex relationship between quantitative MBF, coronary anatomy, revascularization, and outcome in chronic CAD.

\section{Acknowledgements}

Authors acknowledge financial support by grants from the Academy of Finland, the Finnish Foundation for Cardiovascular Research and State Research Funding of Turku University Hospital.

\section{References}

1. Sciagrà R, Lubberink M, Hyafil F, Saraste A, Slart RHJA, Agostini D, et al. EANM procedural guidelines for PET/CT quantitative myocardial perfusion imaging. Eur J Nucl Med Mol Imaging 2021;48:1040-69.

2. Danad I, Uusitalo V, Kero T, Saraste A, Raijmakers PG, Lammertsma AA, et al. Quantitative assessment of myocardial perfusion in the detection of significant coronary artery disease: Cutoff values and diagnostic accuracy of quantitative $[(15) \mathrm{O}] \mathrm{H} 2 \mathrm{O}$ PET imaging. J Am Coll Cardiol 2014;64:1464-75. 
3. Danad I, Raijmakers PG, Driessen RS, Leipsic J, Raju R, Naoum C, et al. Comparison of coronary CT angiography, SPECT, PET, and hybrid imaging for diagnosis of ischemic heart disease determined by fractional flow reserve. JAMA Cardiol 2017;2:1100-7.

4. Danad I, Raijmakers PG, Driessen RS, Leipsic J, Raju R, Naoum $\mathrm{C}$, et al. Comparison of coronary CT angiography, SPECT, PET, and hybrid imaging for diagnosis of ischemic heart disease determined by fractional flow reserve. JAMA Cardiol 2017;2:1100-7.

5. Kajander S, Joutsiniemi E, Saraste M, Pietilä M, Ukkonen H, Saraste A, et al. Cardiac positron emission tomography/computed tomography imaging accurately detects anatomically and functionally significant coronary artery disease. Circulation 2010;122:603-13.

6. Promteangtrong C, Jantarato A, Kunawudhi A, Kiatkittikul P, Siripongsatian D, Boonkawin N, et al. Clinical impact of quantitative [15O] $\mathrm{H} 2 \mathrm{O}$ PET/CT myocardial perfusion imaging on decision-making regarding invasive management of coronary artery disease. J Nucl Cardiol 2021 (in press)

7. Danad I, Raijmakers PG, Harms HJ, van Kuijk C, van Royen N, Diamant $\mathrm{M}$, et al. Effect of cardiac hybrid ${ }^{15} \mathrm{O}$-water PET/CT imaging on downstream referral for invasive coronary angiography and revascularization rate. Eur Heart J Cardiovasc Imaging 2014;15:170-9.

8. Stenström I, Maaniitty T, Uusitalo V, Ukkonen H, Kajander S, Mäki M, et al. Absolute stress myocardial blood flow after coronary CT angiography guides referral to invasive angiography. JACC Cardiovasc Imaging 2019;12:2266-7.

9. Maaniitty T, Stenström I, Bax JJ, Uusitalo V, Ukkonen H, Kajander S, et al. Prognostic value of coronary CT angiography with selective PET perfusion imaging in coronary artery disease. JACC Cardiovasc Imaging 2017;10:1361-70.

10. Bom MJ, van Diemen PA, Driessen RS, Everaars H, Schumacher SP, Wijmenga JT, et al. Prognostic value of [15O]H2O positron emission tomography-derived global and regional myocardial perfusion. Eur Heart J Cardiovasc Imaging 2020;21:777-86.

11. Harjulahti E, Maaniitty T, Nammas W, Stenström I, Biancari F, Bax JJ, et al. Global and segmental absolute stress myocardial blood flow in prediction of cardiac events: [15O] water positron emission tomography study. Eur J Nucl Med Mol Imaging. 2020. h ttps://doi.org/10.1007/s00259-020-05093-2.

12. Liga R, Vontobel J, Rovai D, Marinelli M, Caselli C, Pietila M, et al. Multicentre multi-device hybrid imaging study of coronary artery disease: results from the EValuation of INtegrated Cardiac Imaging for the Detection and Characterization of Ischaemic Heart Disease (EVINCI) hybrid imaging population. Eur Heart J Cardiovasc Imaging 2016;17(9):951-60.

13. Stenström I, Maaniitty T, Uusitalo V, Pietilä M, Ukkonen H, Kajander S, et al. Frequency and angiographic characteristics of coronary microvascular dysfunction in stable angina: A hybrid imaging study. Eur Heart J Cardiovasc Imaging 2017;18:1206-13.

14. Knuuti J, Wijns W, Saraste A, Capodanno D, Barbato E, FunckBrentano C, et al. 2019 ESC Guidelines for the diagnosis and management of chronic coronary syndromes. Eur Heart J 2020;41:407-77.

15. Maron DJ, Hochman JS, Reynolds HR, Bangalore S, O'Brien SM, Boden WE, et al. Initial invasive or conservative strategy for stable coronary disease. N Engl J Med 2020;382:1395-407.

16. Hachamovitch R, Hayes SW, Friedman JD, Cohen I, Berman DS. Comparison of the short-term survival benefit associated with revascularization compared with medical therapy in patients with no prior coronary artery disease undergoing stress myocardial perfusion single photon emission computed tomography. Circulation 2003;107:2900-7.

17. Patel KK, Spertus JA, Chan PS, Sperry BW, Thompson RC, Al Badarin F, et al. Extent of myocardial ischemia on positron emission tomography and survival benefit with early revascularization. J Am Coll Cardiol 2019;74:1645-54.

18. Miller RJH, Bonow RO, Gransar H, Park R, Slomka PJ, Friedman $\mathrm{JD}$, et al. Percutaneous or surgical revascularization is associated with survival benefit in stable coronary artery disease. Eur Heart $\mathbf{J}$ Cardiovasc Imaging 2020;21:961-70.

Publisher's Note Springer Nature remains neutral with regard to jurisdictional claims in published maps and institutional affiliations. 